\title{
Confirming the phylogenetic position of the genus Muscodor and the description of a new Muscodor species
}

\section{Chen $\mathrm{JJ}^{1 \#}$, Feng $\mathrm{XX}^{2 \#}$, Xia CY${ }^{1}$, Kong $\mathrm{DD}^{2}$, Qi $\mathrm{ZY}^{2}$, Liu $\mathrm{F}^{3}$, Chen $\mathrm{D}^{3}$, Lin $\mathrm{FC}^{1}$, Zhang $\mathbf{C L}^{\mathbf{1}^{*}}$}

\author{
${ }^{1}$ State Key Laboratory for Rice Biology, Institute of Biotechnology, Zhejiang University, Hangzhou, Zhejiang, China \\ ${ }^{2}$ Agricultural Experiment Station, Zhejiang University, Hangzhou, Zhejiang, China \\ ${ }^{3}$ Naban River Watershed National Nature Reserve, Jinghong, Yunnan, China
}

Chen JJ, Feng XX, Xia CY, Kong DD, Qi ZY, Liu F, Chen D, Lin FC, Zhang CL 2019 - The phylogenetic position of the genus Muscodor and the description of a new Muscodor species. Mycosphere 10(1), 187-201, Doi 10.5943/mycosphere/10/1/2

\begin{abstract}
It has been suggested that the genus Muscodor should be rejected, while many new species recently introduced are based on chemical profiles of volatile organic compounds and insufficient phylogenetic analyses. The ITS rRNA gene was used for identification of Muscodor species, but has limitations. A four-locus (ITS rRNA, 28S rRNA, RPB2 and TUB1 gene) combined phylogenetic tree has been reconstructed in the current study to confirm that the genus Muscodor is phylogenetically distinct from other closely related genera. A new Muscodor species named Muscodor yunnanensis is described based on phylogenetic analyses and culture characteristics.
\end{abstract}

Key words - 1 new species - Muscodor - phylogenetic position - new species - four-loci

\section{Introduction}

Muscodor is a genus introduced by the discovery of Muscodor albus isolated from branches of Cinnamomum zeylanicum from Honduras (Worapong et al. 2001), which comprises endophytes placed in Xylariales genera incertae sedis (Xylariales, Sordariomycetes, Ascomycota) (Maharachchikumbura et al. 2016, Daranagama et al. 2018) or Sordariomycetes genera incertae sedis (Wijayawardene et al. 2017, 2018). The genus is known for producing volatile organic compounds (VOCs). VOCs produced by Muscodor species can induce lethal effects against a broad range of plant and human fungal pathogens, nematodes and insects (Strobel 2006, Mercier \& Tamezguerra 2007, Kudalkar et al. 2012) and is therefore considered to be a promising agent of myco-fumigation in agriculture and industry (Mitchell et al. 2001, Atmosukarto et al. 2005, Strobel 2006, Suwannarach et al. 2016).

To date, 21 species (Zhang et al. 2010, Meshram et al. 2015, Siri-udom et al. 2016, Roskov et al. 2018) have been recorded in this genus based on culture characteristics, chemical profiles of VOCs and molecular phylogenetic analyses. However, the identification of Muscodor remains ambiguous and the position of this genus remains controversial. There are several reasons for this: 1) Muscodor species are completely sterile as reproductive structures have never been observed in any medium, and these characters are not available for identification. Thus, characterization is only based on colonies and mycelial characteristics, even though some species may exhibit a particular 
hyphal arrangement (Mitchell et al. 2001, Zhang et al. 2010), 2) the profile of unique volatile metabolites produced by Muscodor species analysed through gas chromatography coupled to mass spectrometry (GC-MS) are used as criteria for classification (Strobel 2006, MaríAc et al. 2009), while no other taxa in Xylariales have been compared based on volatile profiles as with Muscodor species (Stadler et al. 2013), 3) for most Muscodor species, ITS rRNA gene sequences are the only molecular phylogenetic data available and the limitations for reconstruction of the phylogenetic relationships within the Xylariales have been discussed (Tang et al. 2009, Stadler et al. 2013).

Since one genus can only have one name (Hawksworth 2011, Gams et al. 2012a, b, Hawksworth 2012), the new nomenclature calls for abandoning some ill-defined asexual morph genera including Muscodor. Stadler et al. (2013) doubted the definition of Muscodor, but did not advise to integrate the younger asexual morph genus Muscodor into any of the older genera. Wendt et al. (2018) even suggested to reject the genus for not following good taxonomic standards as presented in Stadler et al. (2013). Therefore, in the current study, we attempt to clarify the classification status of Muscodor though further identified materials and a more appropriate multigene genealogy. During our investigation of the endophytic fungi in some gramineous plants in the southwest of China, we found nine strains belonging to the genus Muscodor and one, numbered W-S-38, is described as a novel Muscodor species, named Muscodor yunnanensis according to multi-loci phylogenetic evidence. These nine strains, along with 13 strains including Muscodor fengyangensis (Zhang et al. 2010) were analysed by single ITS rRNA gene, RPB2 gene and fourloci (ITS rRNA, 28S rRNA, RPB2 and TUB1 genes) combined phylogenies, which demonstrate the phylogenetic position of Muscodor.

\section{Materials \& Methods}

\section{Isolation of fungi}

The grasses were sampled during August 2015 from the Naban River Watershed National Natural Reserve (E $100^{\circ} 32^{\prime}-100^{\circ} 44^{\prime}$, N 22 $04^{\prime}-22^{\circ} 17^{\prime}$ ) in Yunnan Province of China, which is one of biodiversity hot spots of the world (Shen et al. 2017). The samples were packed into a box with ice. Within 48 hours after sampling, healthy roots, stems and leaves were rinsed first with tap water, then sterilized with $75 \%$ ethanol for 5 minutes, followed by immersion into $1 \%$ sodium hypochlorite $(\mathrm{NaOCl})$ for 10 minutes and finally rinsed again three times with sterile-distilled water. The plant tissues were cut into about $5 \mathrm{~mm}$ long segments and every ten segments of each part were placed on a plate of potato dextrose agar (PDA) supplemented with $50 \mathrm{mg} / \mathrm{L}^{-1}$ chloramphenicol to prevent bacterial growth. The plates were then incubated at $25^{\circ} \mathrm{C}$ in darkness and observed every 12 hours and arising fungal cultures transferred to fresh PDA plates for sub-culturing.

\section{Morphology}

The cultures were incubated on PDA at $25{ }^{\circ} \mathrm{C}$ in darkness for growth rates measurements every day and culture characteristics observations.

\section{Scanning electron microscopy (SEM) analysis}

For SEM analysis, the fungal strain was incubated on PDA at $25^{\circ} \mathrm{C}$ in darkness for 7 days. The peripheral front of the radial culture was then carefully removed with a scalpel. SEM was performed using HITACHI SU8000 microscope.

\section{Fungal DNA extraction, PCR and sequencing}

Seven days old colonies grown on PDA were used for genomic DNA extraction following the protocol of Cubero et al. (1999). ITS rRNA gene was sequenced for nine strains isolated in current study and three loci including 28S rRNA, RPB2 and TUB1 genes were sequenced for 5 selected strains as ITS rRNA gene sequences of strains $\mathrm{Y}-\mathrm{L}-54, \mathrm{~W}-\mathrm{T}-27$ were same as strains $\mathrm{W}-\mathrm{S}-41, \mathrm{Y}-$ S-35 respectively and ITS rRNA gene sequences of strains $\mathrm{W}-\mathrm{S}-35, \mathrm{Y}-\mathrm{L}-43$ were same as strain $\mathrm{W}-\mathrm{S}-38$. The primers and annealing temperatures used for amplification and sequencing were in 
summarised in Table 1. The PCR products were sequenced in an ABI 3730 sequencer using the same primers as PCR.

Table 1 Primers and annealing temperatures used for amplification and sequencing.

\begin{tabular}{lcclc}
\hline Locus & $\begin{array}{c}\text { Annealing } \\
\text { temp }\left({ }^{\circ} \mathbf{C}\right)\end{array}$ & Cycles & \multicolumn{1}{c}{ Primer and Primer sequence (5'-3') } & Reference \\
\hline ITS & 52 & 35 & $\begin{array}{l}\text { ITS1 TCCGTAGGTGAACCTGCGG } \\
\text { ITS4 TC CTCCGCTTATTGATATGC }\end{array}$ & White et al. 1990 \\
28S & 48 & 35 & $\begin{array}{l}\text { LR0R ACCCGCTGAACTTAAGC } \\
\text { LR5 TCCTGAGGGAAACTTCG }\end{array}$ & White et al. 1990 \\
RPB2 & 48 & 35 & $\begin{array}{l}\text { RPB2-5f GAYGAYMGWGATCAYTTYGG } \\
\text { RPB2-7cr CCCATRGCTTGYTRCCCAT } \\
\text { bena-T1 AACATGCGTGAGATTGTAAGT } \\
\text { bena-T22 TCTGGATGTTGTTGGGAATCC }\end{array}$ & L'Donnell et al. 1997 \\
TUB1 & 48 & 35 & . 1999 \\
\hline
\end{tabular}

\section{Sequence alignment and phylogenetic analyses}

The sequences were subjected BLAST analysis at NCBI and aligned with reference sequences of Muscodor strains using CLUSTAL X 2.1 (Larkin et al. 2007). Isolates used in this study and GenBank accessions are listed in Table 2. The alignment was manually modified in GENEDOC (Nicholas \& Nicholas 1997) to remove extra 5' and 3' sequences from where the sequences were overlapped. DAMBE5 (Xia 2013) was used to perform the substitution saturation test. Phylogenetic analyses were conducted by maximum parsimony (MP), Bayesian inference (BI) and maximum likelihood (ML) analyses using PAUP v.4.0a152 (Swofford 2002), MrBayes v.3.2.6 (Ronquist et al. 2012) and IQ-TREE v.1.6.3 (Nguyen et al. 2015), respectively. MP analysis was performed with heuristic search algorithm (1000 random sequence stepwise additions) with a tree-bisectionreconnection (TBR) branch swapping. Maxtrees were set to 5000, branches of zero length were collapsed and all equally parsimonious trees were saved. The branch support values of MP analysis were calculated using a bootstrapping method with 1000 replicates. BI analysis was using a Markov Chain Monte Carlo (MCMC) algorithm. The best-fit nucleotide substitution model of BI and ML analyses was specified by jModelTest 2.1.7 (Darriba et al. 2012) under default settings followed by Akaike information criterion (AIC). Trees were sampled every 100 generations from 5,000,000 generations resulting in total 50,000 trees. The first 12,500 trees (first $25 \%$ samples by default in the software) were discarded representing the burn-in phase and the remaining 37,500 trees were for calculating posterior probabilities (PP) values in the majority rule consensus tree. In ML analysis, we obtained branch supports with the ultrafast bootstrap (Hoang et al. 2017) implemented in the IQTREE. The p-distance was calculated by MEGA version 6 (Tamura et al. 2013) with substitutions: Transitions + Transversions.

Table 2 Strains used in this study and GenBank accessions.

\begin{tabular}{lcccc}
\hline \multicolumn{1}{c}{ Strain } & ITS & 28S & RPB2 & Tub1 \\
\hline Annulohypoxylon moriforme var. microdiscum & DQ631935 & DQ840061 & DQ631960 & DQ840095 \\
JF TH-28-01 & DQ223751 & DQ840063 & - & AY951663 \\
Annulohypoxylon nitens ST2313/YMJ & & & & \\
91022108 & AJ390409 & DQ840064 & DQ631962 & - \\
Annulohypoxylon stygium CM AT-010 & EU552098 & - & - & - \\
Anthostomella brabeji & DQ890026 & - & - & - \\
Anthostomella eucalyptorum & EU552101 & - & - & - \\
Anthostomella proteae & AY862571 & - & - & - \\
Astrocystis cocoes & AF405301 & AF382366 & DQ368653 & - \\
Bartalinia robillardoides (outgroup) & DQ631933 & DQ840055 & - & AY951674 \\
Biscogniauxia capnodes CM AT-015/YMJ 142 & DQ & & &
\end{tabular}


Table 2 Continued.

\begin{tabular}{|c|c|c|c|c|}
\hline Strain & ITS & $28 \mathrm{~S}$ & RPB2 & Tub1 \\
\hline Biscogniauxia mediterranea CBS 280.61 & AJ390413 & - & - & AY951684 \\
\hline Biscogniauxia simplicior B73C/YMJ 136 & AJ390416 & - & - & AY951686 \\
\hline Camillea tinctor $\mathrm{C} 83 \mathrm{C}$ & AJ390421 & - & - & - \\
\hline Camillea tinctor $\mathrm{C} 84 \mathrm{M}$ & AJ390422 & - & - & - \\
\hline Collodiscula japonica & JF440974 & - & - & - \\
\hline Creosphaeria sassafras CM AT-018 & DQ631934 & DQ840056 & DQ631964 & - \\
\hline Creosphaeria sassafras $\mathrm{Cr} 90 \mathrm{M}$ & AJ390424 & - & - & - \\
\hline Creosphaeria sassafras $\mathrm{Cr} 91 \mathrm{M}$ & AJ390425 & - & - & - \\
\hline Daldinia concentrica M-0066225 & AY616681 & - & DQ368651 & - \\
\hline Daldinia loculata $\mathrm{HJ} 108$ & AF176959 & - & - & AY951698 \\
\hline Daldinia petriniae $\mathrm{H}$ Knudsen s.n. & AF176970 & - & - & AY951699 \\
\hline Entoleuca mammata ATCC 58108 & AF201713 & - & - & - \\
\hline Entoleuca mammata E.MAMM1 & AJ246232 & - & - & - \\
\hline Entoleuca mammata E.MAMM3 & $\mathrm{AJ} 246235$ & - & - & - \\
\hline Fungal endophyte isolate 1128 & EU686807 & - & - & - \\
\hline Fungal endophyte isolate 1138 & EU686808 & - & - & - \\
\hline Fungal endophyte isolate 1155 & EU686810 & - & - & - \\
\hline Fungal endophyte isolate 1157 & EU686811 & - & - & - \\
\hline Fungal endophyte isolate 1730 & EU686946 & - & - & - \\
\hline Fungal endophyte isolate 1744 & EU686949 & - & - & - \\
\hline Fungal endophyte isolate 2067 & EU687018 & - & - & - \\
\hline Fungal endophyte isolate 2161 & EU687035 & - & - & - \\
\hline Fungal endophyte sp. ECD-2008 & EU686114 & - & - & - \\
\hline Fungal endophyte sp. P1907B & EU977281 & - & - & - \\
\hline Fungal endophyte sp. P913A & EU977208 & - & - & - \\
\hline Fungal sp. R15 & AY699660 & - & - & - \\
\hline Fungal sp. ZH S13-1-2 & GQ220337 & HM034864 & GQ241929 & HM034845 \\
\hline Hypoxylon fragiforme agrD459/HKUCC 1022 & AY616690 & AY083829 & - & - \\
\hline Hypoxylon monticulosum GZ AT-M050 & DQ631936 & DQ840067 & DQ631955 & - \\
\hline Hypoxylon monticulosum HK AT-PTC015 & DQ631939 & - & DQ631950 & DQ840096 \\
\hline Kretzschmaria clavus CBS 826.72 & AJ390435 & - & - & - \\
\hline Kretzschmaria clavus JP 3113 & AJ390434 & - & - & - \\
\hline Kretzschmaria clavus K171C & AJ390437 & - & - & - \\
\hline Muscodor albus & AF324336 & - & - & - \\
\hline Muscodor albus $141-3 \mathrm{~s}$ & AY927993 & - & - & - \\
\hline Muscodor albus 9-6 & HM034857 & HM034865 & KC243321 & HM034844 \\
\hline Muscodor albus aa3 & JN426991 & - & - & - \\
\hline Muscodor albus GP100 & AY555731 & - & - & - \\
\hline Muscodor albus GP115 & AY527048 & - & - & - \\
\hline Muscodor albus GP206 & AY527047 & - & - & - \\
\hline Muscodor albus isolate E-6 & EF183509 & - & - & - \\
\hline Muscodor albus KN26 & AY527044 & - & - & - \\
\hline Muscodor albus KN27 & AY527046 & - & - & - \\
\hline Muscodor albus MFC2 & AY244622 & - & - & - \\
\hline Muscodor albus MOW12 & JX469138 & - & - & - \\
\hline Muscodor albus TP21 & AY527045 & - & - & - \\
\hline Muscodor camphorae 1639CCSTITD & KC481681 & - & - & - \\
\hline
\end{tabular}


Table 2 Continued.

\begin{tabular}{|c|c|c|c|c|}
\hline Strain & ITS & $28 \mathrm{~S}$ & RPB2 & Tub1 \\
\hline Muscodor cinnanomi strain CMU_Cib 461 & GQ848369 & - & - & - \\
\hline Muscodor coffeanum isolate CDA743 & KP862879 & - & KP862880 & - \\
\hline Muscodor coffeanum strain CDA739 & KM514680 & - & - & - \\
\hline Muscodor coffeanum strain CDA741 & KM514681 & - & - & - \\
\hline Muscodor crispans isolate $\mathrm{B}-23$ & EU195297 & - & - & - \\
\hline Muscodor darjeelingensis 1CCSTITD & JQ409997 & - & - & - \\
\hline Muscodor equiseti strain CMU-M2 & JX089322 & - & - & - \\
\hline Muscodor fengyangensis ZJLQ023 & HM034856 & HM034859 & HM034849 & HM034843 \\
\hline Muscodor fengyangensis ZJLQ024 & HM034855 & HM034861 & HM034851 & HM034842 \\
\hline Muscodor fengyangensis ZJLQ070 & HM034853 & HM034858 & HM034847 & HM034841 \\
\hline Muscodor fengyangensis ZJLQ151 & HM034852 & HM034860 & HM034848 & HМ034839 \\
\hline Muscodor fengyangensis ZJLQ374 & HM034854 & HМ034862 & HM034850 & HM034840 \\
\hline Muscodor ghoomensis 6CCSTITD & KF537625 & - & - & - \\
\hline Muscodor heveae RTM5-IV3 & KF850712 & - & - & - \\
\hline Muscodor indicus 6(b)CCSTITD & KF537626 & - & - & - \\
\hline Muscodor kashayum 16AMLWLS & KC481680 & - & - & - \\
\hline Muscodor musae strain CMU-MU3 & JX089323 & - & - & - \\
\hline Muscodor musae strain ORE8 & KR011970 & - & - & - \\
\hline Muscodor oryzae strain CMU-WR2 & JX089321 & - & - & - \\
\hline Muscodor roseus A3-5 & AH010859 & - & - & - \\
\hline Muscodor sp. 16AMLWLS & KC481680 & - & - & - \\
\hline Muscodor sp. 1CCSTITD & JQ409997 & - & - & - \\
\hline Muscodor sp. 2CCSTITD & JQ409998 & - & - & - \\
\hline Muscodor sp. 3_4_3 & KF269183 & - & - & - \\
\hline Muscodor sp. A3-5 & AY034665 & - & - & - \\
\hline Muscodor sp. AB-2011 & JN426991 & - & - & - \\
\hline Muscodor sp. CA-01 & KF229758 & - & - & - \\
\hline Muscodor sp. CMU20 & GQ924909 & - & - & - \\
\hline Muscodor sp. D31 & EF564148 & - & - & - \\
\hline Muscodor sp. E6710b & HM999898 & - & - & - \\
\hline Muscodor sp. E8514i & HQ117854 & - & - & - \\
\hline Muscodor sp. EXH1_22 & KF227856 & - & - & - \\
\hline Muscodor sp. Fun50W1 & KF496182 & - & - & - \\
\hline Muscodor sp. Fun56W3 & KF496186 & - & - & - \\
\hline Muscodor sp. GBA & GU797134 & - & - & - \\
\hline Muscodor sp. GS3_3_4 & KF128788 & - & - & - \\
\hline Muscodor sp. GS5_3_5 & KF269187 & - & - & - \\
\hline Muscodor sp. GSH5_3_5 & KF128812 & - & - & - \\
\hline Muscodor sp. GSM5_5_7 & KF128852 & - & - & - \\
\hline Muscodor sp. M112 & HM595541 & MG866043 & HM595625 & MG866071 \\
\hline Muscodor sp. M153 & MG866055 & MG866044 & MG866062 & MG866072 \\
\hline Muscodor sp. M21 & HM595540 & MG866041 & HM595624 & MG866069 \\
\hline Muscodor sp. M211 & MG866056 & MG866045 & MG866063 & MG866073 \\
\hline Muscodor sp. M25 & HM595539 & MG866042 & HM595623 & MG866070 \\
\hline Muscodor sp. M7 & EF564149 & - & - & - \\
\hline Muscodor sp. N128 & KP689118 & - & - & - \\
\hline Muscodor sp. N190 & KP689119 & - & - & - \\
\hline
\end{tabular}


Table 2 Continued.

\begin{tabular}{|c|c|c|c|c|}
\hline Strain & ITS & $28 S$ & RPB2 & Tub1 \\
\hline Muscodor sp. N28 & EF564150 & - & - & - \\
\hline Muscodor sp. OM-01 & KF229762 & - & - & - \\
\hline Muscodor sp. RTM5-IV1 & KF850710 & - & - & - \\
\hline Muscodor sp. RTM5-IV2 & KF850711 & - & - & - \\
\hline Muscodor sp. RTM5-IV4 & KF850713 & - & - & - \\
\hline Muscodor sp. S18-3v1 & EU636700 & HМ034863 & FJ480346 & HM034846 \\
\hline Muscodor sp. SR-2011 & JF938595 & - & - & - \\
\hline Muscodor sp. UBSX & KJ425599 & - & - & - \\
\hline Muscodor sp. UFMGCB 4666 & JN031052 & - & - & - \\
\hline Muscodor sp. VC-01 & KF229754 & - & - & - \\
\hline Muscodor sp. WG-2009a & FJ664551 & - & - & - \\
\hline Muscodor strobelii 6610CMSTITBRT & JQ409999 & - & - & - \\
\hline Muscodor suthepensis strain CMU462 & JN558830 & - & - & - \\
\hline Muscodor sutura SR-2011 & JF938595 & - & - & - \\
\hline Muscodor tigerii 2CCSTITD & JQ409998 & - & - & - \\
\hline Muscodor vitigenus & AY100022 & - & - & - \\
\hline Muscodor yucatanensis strain B110 & FJ917287 & - & - & - \\
\hline Muscodor yunnanensis $\mathrm{W}-\mathrm{S}-38$ & MG866046 & MG866038 & MG866059 & MG866066 \\
\hline Nemania aenea JF 02118 & - & DQ840070 & DQ631951 & DQ840085 \\
\hline Nemania diffusa (FR) FR AT-113 & DQ658238 & DQ840073 & DQ631947 & DQ840088 \\
\hline Nemania serpens FR AT-114 & DQ631942 & DQ840075 & DQ631948 & DQ840086 \\
\hline $\mathrm{N}-\mathrm{L}-7$ & MG866054 & MG866040 & MG866061 & MG866068 \\
\hline Rosellinia corticium GZ-AT-F004 & DQ631940 & DQ840078 & - & DQ840091 \\
\hline Stilbohypoxylon quisquiliarum CM AT-016 & DQ631937 & DQ840079 & - & - \\
\hline Uncultured Sordariomycetidae clone D11 & DQ273343 & - & - & - \\
\hline Whalleya microplaca $\mathrm{W} 80 \mathrm{M}$ & AJ390419 & - & - & - \\
\hline Whalleya microplaca $\mathrm{W} 81 \mathrm{M}$ & AJ390420 & - & - & - \\
\hline $\mathrm{W}-\mathrm{S}-35$ & MG866052 & - & - & - \\
\hline $\mathrm{W}-\mathrm{S}-41$ & MG866047 & MG866036 & MG866057 & MG866064 \\
\hline $\mathrm{W}-\mathrm{T}-27$ & MG866051 & - & - & - \\
\hline Xylaria grammica XT09009 & DQ631944 & DQ840081 & DQ631956 & DQ840090 \\
\hline Xylaria hypoxylon CBS 499.80 & AJ309350 & U47841 & DQ368652 & - \\
\hline Xylaria sp. XT09003 & DQ631945 & DQ840080 & DQ631953 & - \\
\hline Xylariaceae sp. IZ-1249 & AM921731 & - & - & - \\
\hline Xylariaceae sp. JF TH-06-04 & DQ631943 & DQ840069 & DQ631954 & DQ840097 \\
\hline $\mathrm{Y}-\mathrm{L}-43$ & MG866053 & - & - & - \\
\hline $\mathrm{Y}-\mathrm{L}-54$ & MG866049 & - & - & - \\
\hline $\mathrm{Y}-\mathrm{L}-56$ & MG866048 & MG866039 & MG866060 & MG866067 \\
\hline $\mathrm{Y}-\mathrm{S}-35$ & MG866050 & MG866037 & MG866058 & MG866065 \\
\hline
\end{tabular}

\section{Results}

\section{Phylogenetic position of Muscodor}

The substitution saturation test performed by DAMBE5 showed that the substitution was unsaturated. Sequence data and substitution models of ITS rRNA gene, RPB2 gene and combined four-loci are showed in Table 3. The trees reconstructed by MP, BI and ML analyses were similar in topology and a tree reconstructed by BI analysis with BI posterior probabilities, MP bootstrap support values and ML ultrafast bootstrap supports are showed in Figs 1, 2, 3. For the ITS 
phylogenetic tree (Fig. 1), nine strains isolated in the current study were included along with other Muscodor species and closely related genera. Muscodor split into three clades (clade A, B and C) and was well separated from other genera with high statistical supports (BI/MP/ML $=1 / 75 / 100$, $\mathrm{BI} / \mathrm{MP} / \mathrm{ML}=1 / 83 / 97$ and BI/MP/ML = 1/99/100). The 22 strains included in all three clades could represent Muscodor using the RPB2 gene and four-loci combined phylogenetic trees. The closest phylogenetic affinities of Muscodor were Fungal endophyte sp. ECD-2008 and Anthostomella proteae, but other Anthostomella species Anthostomella eucalyptorum and Anthostomella brabeji clustered in another distant clade, while A. brabeji and A. proteae clustered as singletons and were separated with other Anthostomella species (Daranagama et al. 2015). Moreover, the phylogenetic relationships among Muscodor species were not satisfactory, because different species clustered in the same clade like Muscodor oryzae, Muscodor crispans and Muscodor musae, and same species split into different clades, especially in clade A, such as Muscodor albus.

The RPB2 gene phylogenetic tree in current study (Fig. 2) demonstrated the monophyly of Muscodor and the inclusion of three clades (clade A, B and C) with high statistical support $(\mathrm{BI} / \mathrm{MP} / \mathrm{ML}=1 / 100 / 100, \mathrm{BI} / \mathrm{MP} / \mathrm{ML}=1 / 100 / 96$ and $\mathrm{BI} / \mathrm{MP} / \mathrm{ML}=1 / 100 / 100)$. The clade of Muscodor was well separated from the closest clade containing the genera Nemania and Xylaria with high support $(\mathrm{BI} / \mathrm{MP} / \mathrm{ML}=1 / 100 / 100)$. Because of a lack of RPB2 sequence data for most of Muscodor species, only one extra species, Muscodor coffeanum strain CDA739 was added. Consistent with the ITS phylogenetic tree, Muscodor coffeanum strain CDA739 clustered in clade B falling between Muscodor sp. S18-3-1 and Muscodor sp. M25.

The four-loci combined phylogenetic tree (Fig. 3) confirmed the phylogenetic position and the three clades (clade A, B and C) of species of Muscodor with high statistical support $(\mathrm{BI} / \mathrm{MP} / \mathrm{ML}=1 / 100 / 100, \mathrm{BI} / \mathrm{MP} / \mathrm{ML}=1 / 100 / 100$ and BI/MP/ML $=1 / 100 / 100)$. The clade of Muscodor was well separated from the closest clade containing genera Nemania, Stilbohypoxylon, Rosellinia and Xylaria with high support (BI/MP/ML = 1/100/100). In conclusion, the single ITS gene can identify a strain up to the genus Muscodor, but cannot resolve it to species. Moreover, for purpose of the accurate phylogenetic analysis, four or more loci should be used to understand the phylogenies of Muscodor species.

\section{Molecular phylogenetic identification of the new Muscodor species}

The ITS rRNA gene phylogenetic tree (Fig. 1) shows that strain W-S-38 formed an independent lineage with high statistical support $(\mathrm{BI} / \mathrm{MP} / \mathrm{ML}=1 / * / 91)$. The four-loci phylogenetic tree (Fig. 3) showed that strain $\mathrm{W}-\mathrm{S}-38$ also formed an independent clade with high statistical support $(\mathrm{BI} / \mathrm{MP} / \mathrm{ML}=1 / 100 / 100)$ and there were sufficient genetic distances (Table 4) (Zhang et al. 2010) among strain $\mathrm{W}-\mathrm{S}-38$ with phylogenetically close species such as Muscodor suthepensis strain CMU462, Muscodor coffeanum strain CDA741 and Muscodor yucatanensis strain B110. As a result, strain $\mathrm{W}-\mathrm{S}-38$ is recognized as a novel species.

\section{Taxonomy}

Muscodor yunnanensis C.L. Zhang, sp. nov.

Fig. 4

Mycobank: MB824602; Facesoffungi Number: FoF04824

Etymology - named by habitat

Sexual morph: undetermined. Asexual morph: unicellular, hyphae, septate, ramose. The width of the mycelium was $0.9-1.7 \mu \mathrm{m}$ and mycelium frequently intertwined, forming rope-like strands.

Culture characteristics - Cultures incubated on PDA at $25{ }^{\circ} \mathrm{C}$ in darkness, with a linear growth rate of $4.29 \mathrm{~mm}$ diam/day in first 7 days and then be slower. Upper colony white, with longitudinal and sunken stripes, then developing zonate and mycelium in the ring collapsing, reverse colony white and the ring obvious; no conidia and sporulation structure were observed under laboratory conditions. 
Holotype - W-S-38, Naban River Watershed National Nature Reserve (E100 $32^{\prime}-100^{\circ} 44^{\prime}$, N22 $\left.{ }^{\circ} 4^{\prime}-22^{\circ} 17^{\prime}\right)$, Yunnan Province, China, from leaf sheath of Oplismenus undulatifolius, Aug. 2015, deposited in China General Microbiological Culture Collection Center (accession number: CGMCC 3.18908). The accession number of GenBank database for ITS rRNA, 28S rRNA, RPB2 and TUB1 genes sequences were MG866046, MG866038, MG866059 and MG866066, respectively. China.

Habitat and distribution - in leaf sheath of Oplismenus undulatifolius, Yunnan province,

Notes - Strain W-S-38 belonging to this novel species was isolated as a plant endophyte. It is differentiated from other Muscodor species by significant phylogenetic support ((BI/MP/ML = 1/100/100) and high p-distances (Table 4). As for culture characteristics, differences with other phylogenetically close Muscodor species were in Table 5.

Table 3 Sequence data and substitution models of ITS rRNA, RPB2 gene and combined four-loci (ITS rRNA, 28S rRNA, RPB2 and TUB1 genes).

\begin{tabular}{lccccc}
\hline Sequence & $\begin{array}{c}\text { Nucleotide } \\
\text { characters } \\
\text { including gaps }\end{array}$ & $\begin{array}{c}\text { Parsimony } \\
\text { informative } \\
\text { sites }\end{array}$ & $\begin{array}{c}\text { Variable and } \\
\text { parsimony } \\
\text { uninformative sites }\end{array}$ & $\begin{array}{c}\text { Constant } \\
\text { sites }\end{array}$ & $\begin{array}{c}\text { Best-fit nucleotide } \\
\text { substitution model for } \\
\text { BI and ML analyses }\end{array}$ \\
\hline ITS & 566 & 361 & 66 & 139 & TIM3ef+I+G \\
RPB2 & 891 & 427 & 43 & 421 & TIM2+G \\
$\begin{array}{l}\text { Combined } \\
\text { four-loci }\end{array}$ & 3735 & 1479 & 331 & 1925 & TIM2+I+G \\
\hline
\end{tabular}

Table 4 p-Distance of nucleotide sites among the four-loci sequences compared between W-S-38 and close species.

\begin{tabular}{lcccc}
\hline & $\begin{array}{c}\text { M. suthepensis } \\
\text { strain CMU462 }\end{array}$ & W-S-38 & $\begin{array}{c}\text { M. coffeanum } \\
\text { strain CDA741 }\end{array}$ & $\begin{array}{c}\text { M. yucatanensis } \\
\text { strain B110 }\end{array}$ \\
\hline $\begin{array}{l}\text { M. suthepensis } \\
\text { strain CMU462 }\end{array}$ & & & & \\
$\begin{array}{l}\text { W-S-38 } \\
\begin{array}{l}\text { M. coffeanum } \\
\text { strain CDA741 }\end{array}\end{array}$ & 0.050 & & & \\
$\begin{array}{l}\text { M. yucatanensis } \\
\text { strain B110 }\end{array}$ & 0.066 & 0.084 & & \\
\hline
\end{tabular}

Table 5 Culture characteristic comparison with several phylogenetically close Muscodor species.

\begin{tabular}{|c|c|c|c|c|}
\hline Taxon & Host & $\begin{array}{c}\text { Mycelium pigment } \\
\text { production }\end{array}$ & Colonial morphology & $\begin{array}{c}\text { Mycelial } \\
\text { growth }\end{array}$ \\
\hline M. albus $\mathrm{s}^{\mathrm{a}, \mathrm{b}}$ & $\begin{array}{l}\text { Cinnamomum } \\
\text { zeylanicum }\end{array}$ & White & Felt-like mycelium & Rope-like \\
\hline M. suthepensis ${ }^{\mathrm{c}}$ & $\begin{array}{l}\text { Cinnamomum } \\
\text { bejolghota }\end{array}$ & $\begin{array}{l}\text { White in the dark and } \\
\text { pale pink in the light }\end{array}$ & No description & $\begin{array}{l}\text { Rope-like with } \\
\text { coils structure }\end{array}$ \\
\hline M. yucatanensis ${ }^{\mathrm{d}}$ & $\begin{array}{l}\text { Bursera } \\
\text { simaruba }\end{array}$ & $\begin{array}{l}\text { Whitish and ivory- } \\
\text { white for older } \\
\text { colonies }\end{array}$ & $\begin{array}{l}\text { Flocculose pattern and } \\
\text { reverse uncolored, slightly } \\
\text { funiculose }\end{array}$ & $\begin{array}{l}\text { Rope-like with } \\
\text { coils structure } \\
\text { and swollen cell }\end{array}$ \\
\hline W-S-38 & $\begin{array}{l}\text { Oplismenutls } \\
\text { undulatifolius }\end{array}$ & White & $\begin{array}{l}\text { With longitudinal and } \\
\text { sunken stripes, then } \\
\text { developing zonate and } \\
\text { mycelium in the ring } \\
\text { collapsing, }\end{array}$ & Rope-like \\
\hline
\end{tabular}

${ }^{\mathrm{a}}$ Worapong et al. 2001, ${ }^{\mathrm{b}}$ Ezra et al. 2004, ${ }^{\mathrm{c}}$ Suwannarach et al. 2013, ${ }^{\mathrm{d}}$ González et al. 2009 


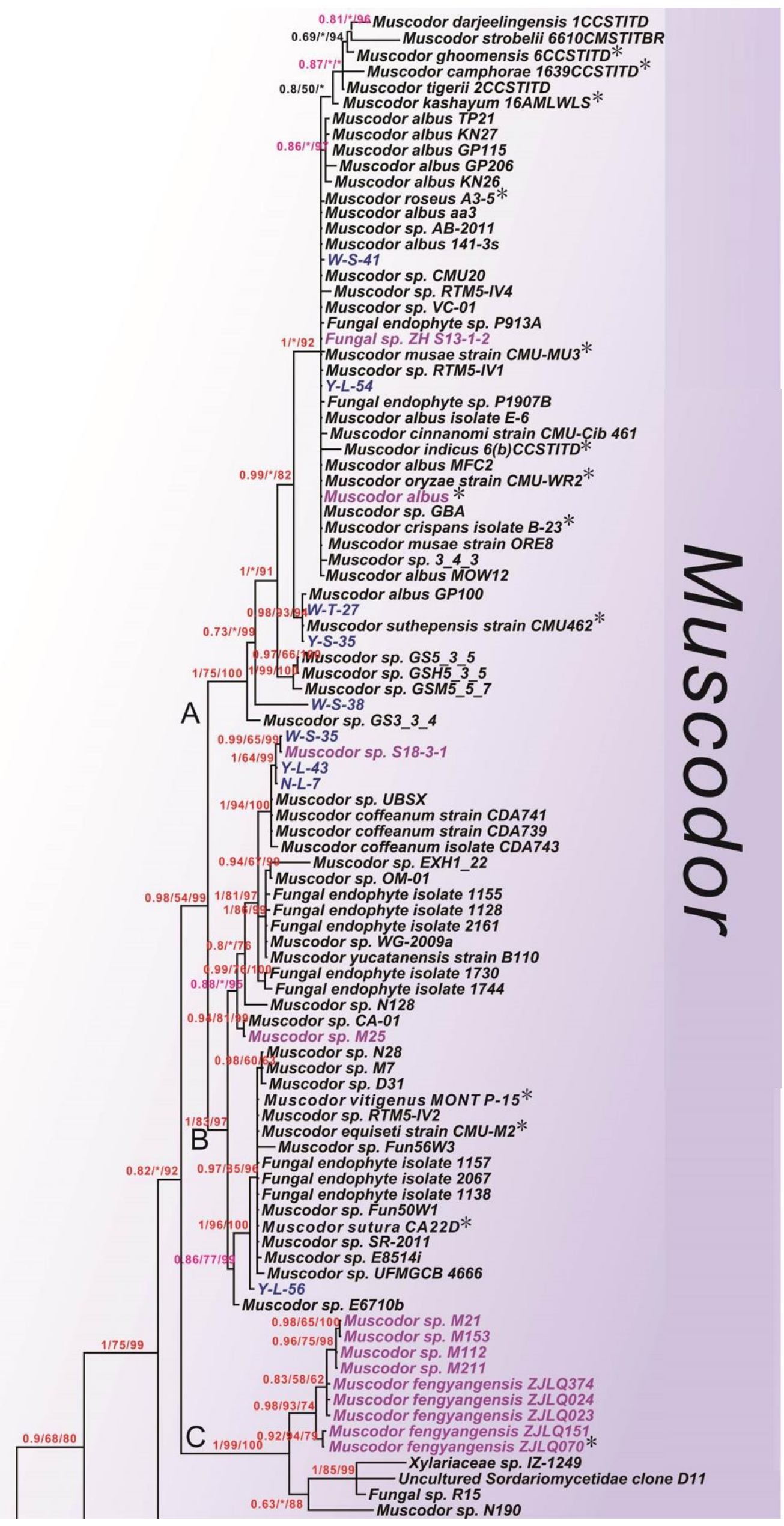




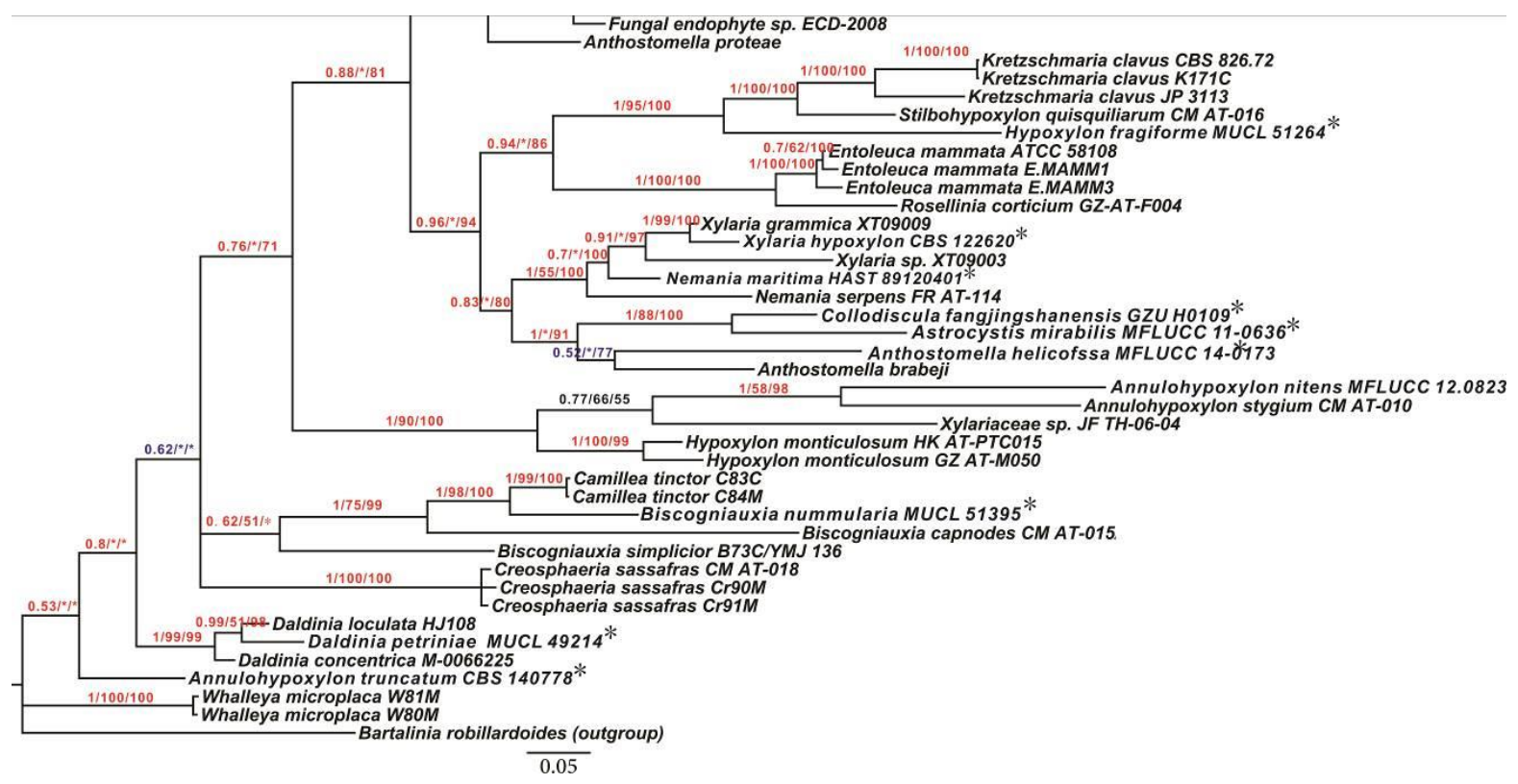

Figure 1 - The phylogenetic tree inferred from MP, BI and ML analyses based on ITS rRNA gene dataset. BI posterior probabilities, MP bootstrap support values and ML ultrafast bootstrap supports are shown at the first, second and third position above branches. Bartalinia robillardoides is defined as an outgroup. * above branches indicates MP and ML phylogenetic tree is not consistent with BI phylogenetic tree here. The bar represents 0.05 substitution per site. ${ }^{*}$ after strains indicates type strains.

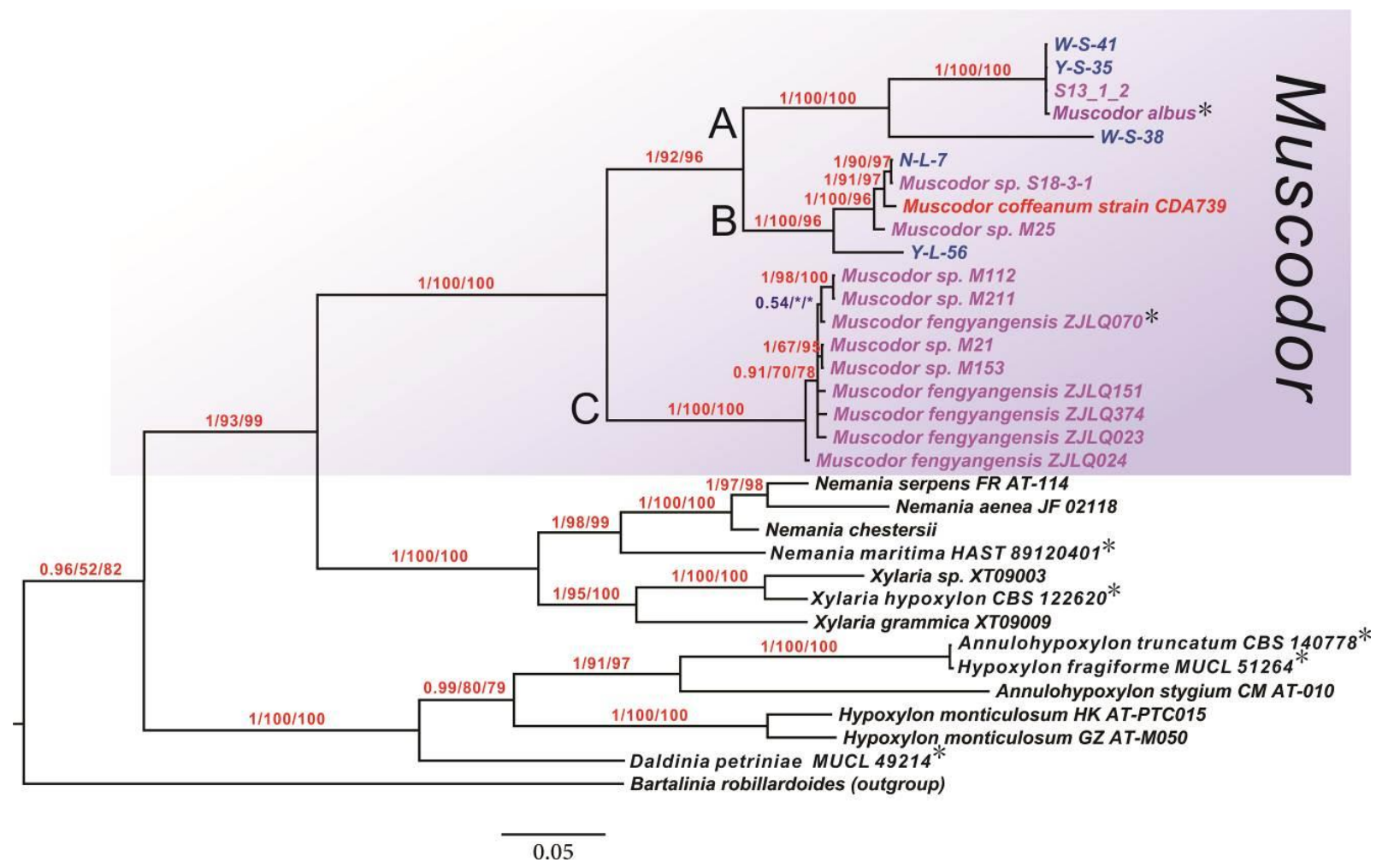

Figure 2 - The phylogenetic tree inferred from MP, BI and ML analyses based on RPB2 gene dataset. BI posterior probabilities, MP bootstrap support values and ML ultrafast bootstrap supports are shown at the first, second and third position above branches. Bartalinia robillardoides is defined as an outgroup. * above branches indicates MP and ML phylogenetic tree is not consistent with BI phylogenetic tree here. The bar represents 0.05 substitution per site. * after strains indicates type strains. 


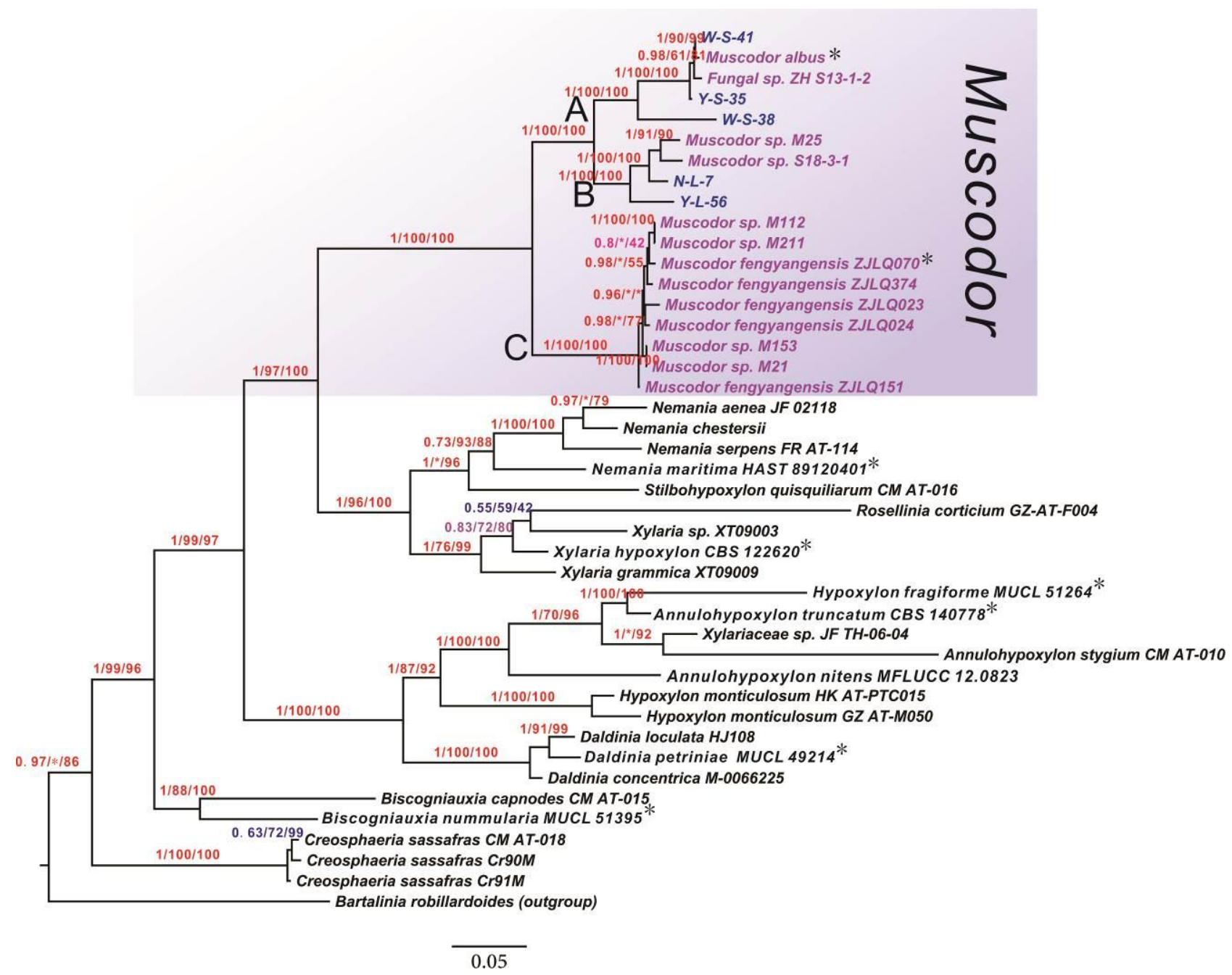

Figure 3 - The phylogenetic tree inferred from MP, BI and ML analyses based on four-loci (ITS rRNA, 28S rRNA, RPB2 and TUB1 genes) dataset. BI posterior probabilities, MP bootstrap support values and ML ultrafast bootstrap supports are shown at the first, second and third position above branches. Bartalinia robillardoides is defined as an outgroup. * above branches indicates MP and ML phylogenetic tree is not consistent with BI phylogenetic tree here. The bar represents 0.05 substitution per site. * after strains indicates type strains.

\section{Discussion}

New nomenclature calls for more accurate identification (Stadler et al. 2013), however, because of the lack of reproductive structures, the identification of Muscodor species mainly relies on phylogenetic analyses and other criteria, such as the differences of their profile of volatiles. For the lack of taxon sampling and reference materials, the rationale that authors used to introduce a new genus appears highly questionable (Stadler et al. 2013). In order to clarify the position of Muscodor, a four-loci phylogenetic tree was reconstructed in this study, which demonstrated that all 18 strains are distributed in three clades in Muscodor that clustered as a monophyletic group. Meshram et al. (2013) separated the genus Muscodor into two clades based on maximum likelihood analysis of ITS1-5.8S-ITS2 region and Siri-udom et al. (2016) separated Muscodor into three major clades based on maximum parsimony analysis of ITS1-5.8S rDNA-ITS2 region. The phylogenetic trees in this study (Figs 1, 2, 3) were topologically similar with the tree in Siri-udom et al. (2016) and the three separate clades were also consistent. The result should be convincing although some sequences in four-loci data were lacking and majority of Muscodor species were sequenced only by ITS.

The p-distance is the proportion (p) of nucleotide sites at which two sequences being compared are different. Zhang et al. (2010) used this approach to determine genetic relatedness of 
isolates, as the p-distance among the five novel Muscodor fengyangensis isolates was the level of millesimal or lower and was always 10- to 20-fold lower than that between Muscodor spp. where sequences for all named Muscodor spp. available was in the same range of the level of hundredth. Saxena et al. (2015) also used p-distance to distinguish Muscodor tigerii within Sordariomycetes, Xylaria and Muscodor spp. In the present study, p-distance of nucleotide sites among the four-loci sequences between W-S-38 and similar species was in the same range as that between Muscodor spp. and was in the level of hundredth, which provided strong evidence to demonstrate that W-S-38 is a novel species.

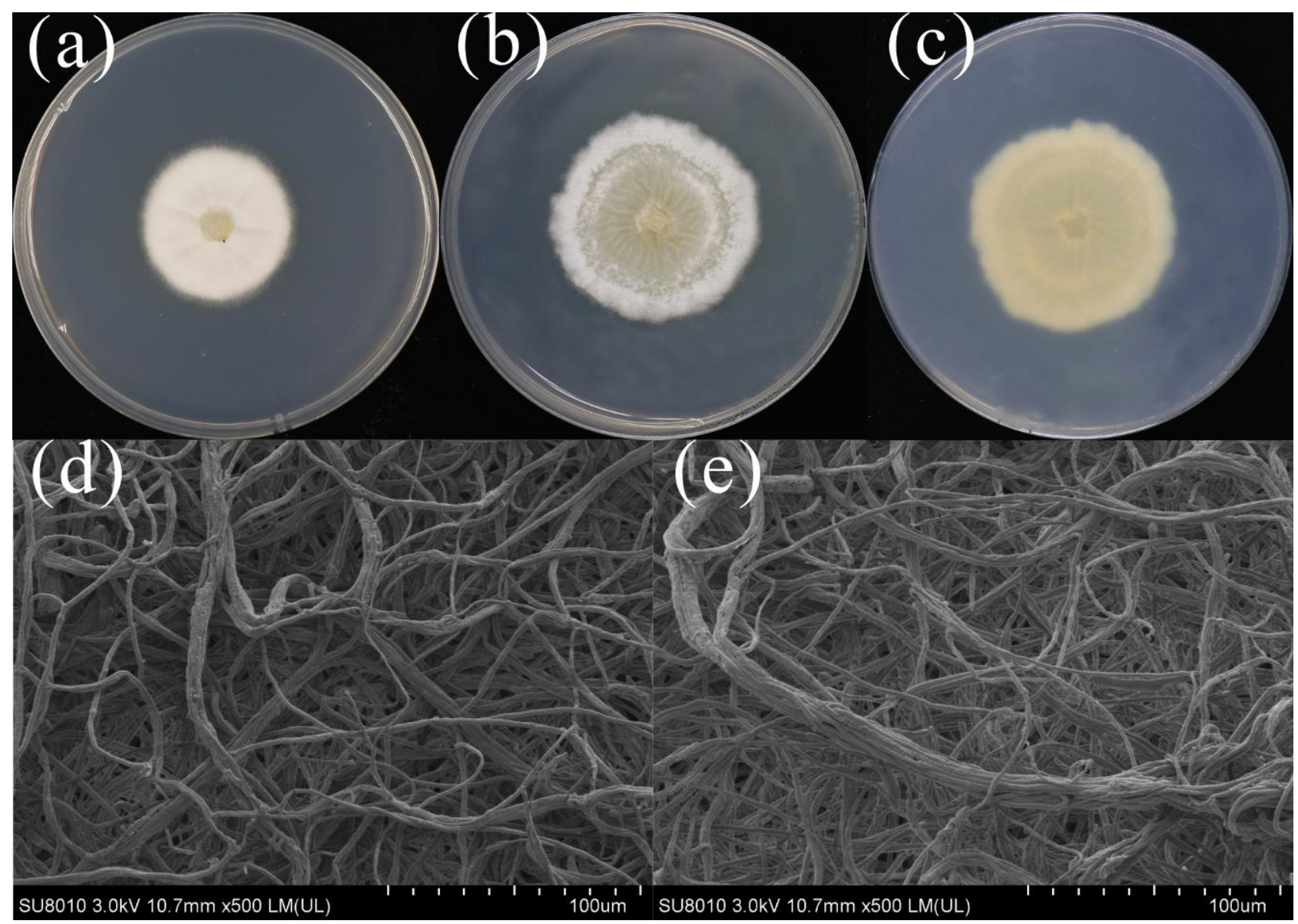

Figure 4 - Morphological characteristics of Muscodor yunnanensis. a colony of strain $\mathrm{W}-\mathrm{S}-38$ on PDA after $7 \mathrm{~d}$ at $25^{\circ} \mathrm{C}$ in darkness. b colony of W-S-38 on PDA after $30 \mathrm{~d}$ at $25^{\circ} \mathrm{C}$ in darkness. c reverse colony of W-S-38 on PDA after $30 \mathrm{~d}$ at $25{ }^{\circ} \mathrm{C}$ in darkness. d scanning electron micrograph of the mycelial arrangement and morphology. e scanning electron micrograph of the fused rope-like hyphae.

There have been decades that ITS rRNA gene was used for diagnostics and molecular phylogenetic identifications in fungi (White et al. 1990, Henrion et al. 1992) and has been proved to be effective in a large quantity of taxonomic groups, and was also proposed to be a universal DNA barcode marker for fungi (Begerow et al. 2010, Schoch et al. 2012). Nonetheless, there were limitations in some aspects and use of only ITS rRNA gene sequences to infer phylogenetic relationships of Xylariales genera incertae sedis was found to be inappropriate (Mazzaglia et al. 2001, Suwannasai et al. 2005, Peláez et al. 2008, Tang et al. 2009, Stadler et al. 2013). More taxa included did not result in better phylogenetic resolution and some taxa could not be well separated (Tang et al. 2009, Stadler et al. 2013). It was also reflected in the current study that Muscodor species could not be separated well from each other at some level and the genera Hypoxylon and Annulohypoxylon were not well separated (Fig. 1). 
In addition, some protein-coding genes such as RPB2 (Liu et al. 1999) were used for phylogeny. In some cases, RPB2 gene provided the higher proportion of informative characters and the phylogeny based on it appeared to be highly resolved (Stadler et al. 2013). Moreover, when RPB2 gene was analysed in combination with other genes, such as with ITS rRNA gene, the phylogenetic tree obtained would be more reliable. Therefore, RPB2 gene would be a promising candidate for identification within Xylariales genera incertae sedis.

\section{Acknowledgements}

This work was supported by The National Key Research and Development Program of China (Grant No. 2016YFD0300706) to Chu-long Zhang and National Key Technology Research and

Development Program of the Ministry of Science and Technology of China (Grant No. 2015BAC02B03) to Fu-cheng Lin. We would express appreciations to the staff of Naban River Watershed National Nature Reserve for collecting samples.

\section{References}

Atmosukarto I, Castillo U, Hess WM, Sears J, Strobel G. 2005 - Isolation and characterization of Muscodor albus I-41.3s, a volatile antibiotic producing fungus. Plant Science 169, 854-861.

Begerow D, Nilsson H, Unterseher M, Maier W. 2010 - Current state and perspectives of fungal DNA barcoding and rapid identification procedures. Applied Microbiology and Biotechnology 87, 99-108.

Cubero E, Sherer EC, Leque FJ, Orozco M, Laughton CA. 1999 - Observation of spontaneous base pair breathing events in the molecular dynamics simulation of a difluorotoluene-containing DNA oligonucleotide. Journal of the American Chemical Society 121, 8653-8654.

Daranagama DA, Camporesi E, Tian Q, Liu X et al. 2015 - Anthostomella is polyphyletic comprising several genera in Xylariaceae. Fungal Diversity 73, 203-238.

Daranagama DA, Hyde KD, Sir EB, Thambugala KM et al. 2018 - Towards a natural classification and backbone tree for Graphostromataceae, Hypoxylaceae, Lopadostomataceae and Xylariaceae. Fungal Diversity 88, 1-165.

Darriba D, Taboada GL, Doallo R, Posada D. 2012 - jModelTest 2: more models, new heuristics and parallel computing. Nature Methods 9, 772-772.

Ezra D, Hess WM, Strobel GA. 2004 - New endophytic isolates of Muscodor albus, a volatile antibiotic producing fungus. Microbiology 150, 4023- 4031.

Gams W, Baral HO, Jaklitsch WM, Kirschner R, Stadler M. 2012a - Clarifications needed concerning the new Article 59 dealing with pleomorphic fungi. IMA Fungus 3, 175-177.

Gams W, Humber RA, Jaklitsch W, Kirschner R, Stadler M. 2012b - Minimizing the chaos following the loss of Article 59: Suggestions for a discussion. Mycotaxon 119, 495-507.

González MC, Anaya AL, Glenn AE, Macías-Rubalcava ML et al. 2009 - Muscodor yucatanensis, a new endophytic ascomycete from Mexican chakah, Bursera simaruba. Mycotaxon 110, 363-372.

Hoang DT, Chernomor O, Von HA, Minh BQ et al. 2017 - UFBoot2: Improving the ultrafast bootstrap approximation. Molecular Biology \& Evolution 35, 518-522.

Hawksworth DL. 2011 - A new dawn for the naming of fungi: impacts of decisions made in Melbourne in July 2011 on the future publication and regulation of fungal names. IMA Fungus 2, 155-162.

Hawksworth DL. 2012 - Managing and coping with names of pleomorphic fungi in a period of transition1, 2. IMA Fungus 3, 15-24.

Henrion B, Tacon FL, Martin F. 1992 - Rapid identification of genetic variation of ectomycorrhizal fungi by amplification of ribosomal RNA genes. New Phytologist 122, 289-298.

Kudalkar P, Strobel G, Riyaz-Ul-Hassan S, Geary B, Sears J. 2012 - Muscodor sutura, a novel endophytic fungus with volatile antibiotic activities. Mycoscience 53, 319-325. 
Larkin MA, Blackshields G, Brown NP, Chenna R et al. 2007 - Clustal W and Clustal X version 2.0. Bioinformatics 23, 2947-2948.

Liu YJ, Whelen S, Hall BD. 1999 - Phylogenetic relationships among ascomycetes: evidence from an RNA polymerse II subunit. Molecular Biology \& Evolution 16, 1799.

Maharachchikumbura SSN, Hyde KD, Jones EBG, Mckenzie EHC et al. 2016 - Families of Sordariomycetes. Fungal Diversity 79, 1-317.

MaríAc GL, Analuisa A, Anthonye G, Marthal MA et al. 2009 - Muscodor yucatanensis, a new endophytic ascomycete from Mexican chakah, Bursera simaruba. Mycotaxon 110, 363-372.

Mazzaglia A, Anselmi N, Vicario S, Vannini A. 2001 - Sequence analysis of the 5.8S rDNA and ITS regions in evaluating genetic relationships among some species of Hypoxylon and related genera. Mycological Research 105, 670-675.

Mercier J, Santamaria JIJ, Guerra PT. 2007 - Development of the volatile-producing fungus Muscodor albus Worapong, Strobel \& Hess as a novel antimicrobial biofumigant. Revista Mexicana De Fitopatología 25, 173-179.

Meshram V, Kapoor N, Saxena S. 2013 - Muscodor kashayum sp. nov. - a new volatile antimicrobial producing endophytic fungus. Mycology 4, 196-204.

Meshram V, Gupta M, Saxena S. 2015 - Muscodor tigerii sp. nov. - Volatile antibiotic producing endophytic fungus from the Northeastern Himalayas. Annals of Microbiology 65, 47-57.

Mitchell AM, Strobel GA, Moore E, Robison R, Sears J. 2001 - Volatile antimicrobials from Muscodor crispans, a novel endophytic fungus. Microbiology 156, 270-277.

Nguyen LT, Schmidt HA, Haeseler A von, Minh BQ. 2015 - IQ-TREE: A fast and effective stochastic algorithm for estimating maximum likelihood phylogenies. Molecular Biology \& Evolution 32, 268-274.

Nicholas K, Nicholas H. 1997 - GeneDoc: a tool for editing and annotating multiple sequence alignments. Distributed by the authors, 4 .

O’Donnell K, Cigelnik E, Weber NS, Trappe JM. 1997 - Phylogenetic relationships among ascomycetous truffles and the true and false morels inferred from $18 \mathrm{~S}$ and $28 \mathrm{~S}$ ribosomal DNA aequence analysis. Mycologia 89, 48-65.

Peláez F, Platas G, Sánchez-Ballesteros J. 2008 - Molecular phylogenetic studies within the Xylariaceae based on ribosomal DNA sequences. Fungal Diversity 31, 111-134.

Ronquist F, Teslenko M, Mark PVD, Ayres DL et al. 2012 - MrBayes 3.2: efficient Bayesian phylogenetic inference and model choice across a large model space. Systematic Biology 61, 539-542.

Roskov Y, Abucay L, Orrell T, Nicolson D et al. 2018 - Species 2000 \& ITIS Catalogue of Life, 26th February 2018. Digital resource at www.catalogueoflife.org/col. Species 2000: Naturalis, Leiden, the Netherlands. ISSN 2405-8858.

Saxena S, Meshram V, Kapoor N. 2015 - Muscodor tigerii sp. nov.-Volatile antibiotic producing endophytic fungus from the Northeastern Himalayas. Annals of Microbiology 65, 47-57.

Schoch CL, Seifert KA, Huhndorf S, Robert V et al. 2012 - Nuclear ribosomal internal transcribed spacer (ITS) region as a universal DNA barcode marker for Fungi. Proceedings of the National Academy of Sciences of the United States of America 109, 6241-6246.

Shen S, Xu G, Li D, Clements DR et al. 2017 - Agrobiodiversity and in situ conservation in ethnic minority communities of Xishuangbanna in Yunnan province, southwest China. Journal of Ethnobiology \& Ethnomedicine 13, 28.

Siri-udom S, Suwannarach N, Lumyong S. 2016 - Existence of Muscodor vitigenus, M. equiseti and $M$. heveae sp. nov. in leaves of the rubber tree (Hevea brasiliensis Müll.Arg.), and their biocontrol potential. Annals of Microbiology 66, 437-448

Stadler M, Kuhnert E, Peršoh D, Fournier J. 2013 - The Xylariaceae as model example for a unified nomenclature following the "One Fungus-One Name" (1F1N) concept. Mycology 4, $5-21$.

Strobel G. 2006 - Harnessing endophytes for industrial microbiology. Current Opinion in Microbiology 9, 240-244. 
Suwannarach N, Kumla J, Bussaban B, Hyde KD et al. 2013 - Molecular and morphological evidence support four new species in the genus Muscodor from northern Thailand. Annals of Microbiology 63, 1341-1351.

Suwannarach N, Bussaban B, Nuangmek W, Pithakpol W et al. 2016 - Evaluation of Muscodor suthepensis strain CMU-Cib462 as a postharvest biofumigant for tangerine fruit rot caused by Penicillium digitatum. Journal of the Science of Food and Agriculture 96, 339-345.

Suwannasai N, Rodtong S, Thienhirun S, Ajs W. 2005 - New species and phylogenetic relationships of Hypoxylon species found in Thailand inferred from the internal transcribed spacer regions of ribosomal DNA sequences. Mycotaxon 94, 303-324.

Swofford DL. 2002 - PAUP*. Phylogenetic analysis using parsimony (*and other methods). Version 4.0b10. Sunderland, Massachusetts: Sinauer Associates.

Tamura K, Stecher G, Peterson D, Filipski A, Kumar S. 2013 - MEGA6: Molecular Evolutionary Genetics Analysis Version 6.0. Molecular Biology and Evolution 30, 2725-2729.

Tang A, Jeewon R, Hyde KD. 2009 - A re-evaluation of the evolutionary relationships within the Xylariaceae based on ribosomal and protein-coding gene sequences. Fungal Diversity 34, $127-155$.

Wendt L, Sir EB, Kuhnert E, Heitkamper S et al. 2018 - Resurrection and emendation of the Hypoxylaceae, recognised from a multigene phylogeny of the Xylariales. Mycological Progress 17, 115-154.

White TJ, Bruns TD, Lee SB, Taylor JW et al. 1990 - Amplification and direct sequencing of fungal ribosomal RNA genes for phylogenetics. PCR Protocols 38, 315-322.

Wijayawardene NN, Hyde KD, Rajeshkumar KC, Hawksworth DL et al. 2017 - Notes for genera: Ascomycota. Fungal Diversity 86, 1-594.

Wijayawardene NN, Hyde KD, Lumbsch HT, Liu JK et al. 2018 - Outline of Ascomycota: 2017. Fungal Diversity 88, 167-263.

Worapong J, Strobel G, Ford EJ, Li JY et al. 2001 - Muscodor albus anam. gen. et sp. nov., an endophyte from Cinnamomum zeylanicum. Mycotaxon 79, 67-79.

Xia X. 2013 - DAMBE5: A comprehensive software package for data analysis in molecular biology and evolution. Molecular Biology \& Evolution 30, 1720.

Zhang CL, Wang GP, Mao LJ, Komon-Zelazowska M et al. 2010 - Muscodor fengyangensis sp. nov. from southeast China: morphology, physiology and production of volatile compounds. Fungal Biology 114, 797-808. 